\title{
ON PARASTROPHIC ALGEBRAS*
}

\author{
BY \\ JAMES BYRNIE SHAW
}

The following paper is a consideration of the relation to a given algebra of algebras produced from it by the interchange of certain of the multiplication constants for an assigned basis. In particular the process of multiplying parastrophically is studied somewhat, with some simple applications.

A very simple test is thus furnished for ascertaining whether a given algebra is semi-simple or not, a test not always applicable but very simple when it is applicable. The parastrophic algebra is not an invariant of the given algebra but changes with a change of the basis. However certain expressions furnished by the parastrophic algebra are invariant for the given algebra, at least in a certain defined sense.

The parastrophic algebras are shown to be determinable when an operator $\rho^{\prime}$ is determined, defined uniquely by $\rho$. In the important case (there are also others) of a Dedekind algebra in canonical form, the operator $\rho^{\prime}$ corresponds to the multiplication by a definite number of the algebra. The parastrophic multiplication becomes then the original multiplication not by $\rho$ but by a definite number $\tau$ of the algebra determined by $\rho$.

The results of much of the paper by Frobenius $\dagger$ on hypercomplex numbers are implied in the parastrophic multiplications and arrived at in a very direct and simple manner. The introduction of the numbers $\kappa$ with their significance indicates the manner of procedure in the reduction of an algebra to its canonical form. $\ddagger$

1. We assume an algebra with a given multiplication table, the products being linear in the variables implied in the numbers of the algebra. Related to this multiplication will be other multiplication tables which we proceed to define. These are chosen from an infinite variety of other related tables on account of their simplicity. Each table defines an algebra which may or may not agree with the original algebra. Thus let two general numbers of these related algebras be

$$
\rho=\sum x_{i} e_{i}, \quad \sigma=\sum y_{j} e_{j},
$$

* Read before the Chicago section of the Society, December 29, 1914.

†Sitzungsberichte K. Akademi e Berlin, 1903, pp. 504-537, 634-645.

$\ddagger$ For terms in general see Shaw, Synopsis of Linear Associative Algebra, 1907. 
wherein the variables $x$ and $y$ run over assigned ranges, not at first specified, and where the units $e$ are any numbers from the algebra, $r$ in number, not subject to any conditional equation of a linear form, such as $\sum a_{i} e_{i}=0$, unless every $a$ vanishes.

We then define the original algebra by the equation of general multiplication

$$
M_{1} \cdot \rho \sigma=\sum x_{i} y_{j} \gamma_{i j k} e_{k} \quad(i, j, k=1, \cdots, r) .
$$

The constants $\gamma$ are assigned in some manner, and may or may not run over the same range as $x$ and $y$. The related algebras that are mentioned here will then be defined by the equations following, and will be named as indicated, the original being the strophic:

$$
\begin{array}{rrr}
M_{2} \cdot \rho \sigma=\sum x_{i} y_{j} \gamma_{j i k} e_{k}, & \text { antistrophic, } \\
M_{3} \cdot \rho \sigma=\sum x_{i} y_{j} \gamma_{i k j} e_{k}, & \text { preparastrophic, } \\
M_{4} \cdot \rho \sigma=\sum x_{i} y_{j} \gamma_{k j i} e_{k}, & \text { postparastrophic, } \\
M_{5} \cdot \rho \sigma=\sum x_{i} y_{j} \gamma_{j k i} e_{k}, & \text { antipostparastrophic, } \\
M_{6} \cdot \rho \sigma=\sum x_{i} y_{j} \gamma_{k i j} e_{k}, & \text { antipreparastrophic. }
\end{array}
$$

The antistrophic is also called the reciprocal. With the exception of the latter, the related algebras are in general not associative, even when the given algebra is associative. For an interpretation of $M_{2}, \cdots, M_{6}$ in terms of operations in the given algebra, see $\S 7$.

2. If we let $\sigma$ be in turn each of the units $e$, we can write out for each of the six algebras a general table of multiplication, consisting of $r$ equations, thus:

$$
\begin{aligned}
M_{1} \cdot \rho e_{j} & =\sum e_{k} X_{j k}^{1}, & X_{j k}^{1} & =\sum x_{i} \gamma_{i j k}, \\
M_{2} \cdot \rho e_{j} & =\sum e_{k} X_{j k}^{2}, & X_{j k}^{2} & =\sum x_{i} \gamma_{j i k}, \\
M_{3} \cdot \rho e_{j} & =\sum e_{k} X_{j k}^{3}, & X_{j k}^{3} & =\sum x_{i} \gamma_{i k j}, \\
M_{4} \cdot \rho e_{j} & =\sum e_{k} X_{j k}^{4}, & X_{j k}^{4} & =\sum x_{i} \gamma_{k j i}, \\
M_{5} \cdot \rho e_{j} & =\sum e_{k} X_{j k}^{5}, & X_{j k}^{5} & =\sum x_{i} \gamma_{j k i}, \\
M_{6} \cdot \rho e_{j} & =\sum e_{k} X_{j k}^{6}, & X_{j k}^{6} & =\sum x_{i} \gamma_{k i j} .
\end{aligned}
$$

3. The number $\rho$ in each case may be looked upon as an operator upon the following number, in fact it may be considered to be a linear substitution on the units. We may arrange the coefficients $X$ in $r$ rows and columns, and form a determinant for each case:

$$
\left|X_{j k}\right| \quad(j, k=1, \cdots, r) .
$$

The column is indicated by the second subscript, the row by the first. This 
determinant we shall indicate by $\Delta^{1}, \Delta^{2}, \Delta^{3}, \Delta^{4}, \Delta^{5}, \Delta^{6}$, in the respective cases, and if we need to indicate the number from which it arises, we shall write $\Delta(\rho)$ with the proper superscript.

4. If we write the multiplication table of each of the algebras as a square array, the multiplicands being the units $e$, arranged in order along the top of the square, and the multipliers the units $e$ arranged in order vertically downwards, along the left side of the square, the product of $e_{j}$ into $e_{k}$ being in the square on the line $j$ and in column $k$, then we have the entry

$$
\begin{array}{lll}
M_{1} \cdot e_{j} e_{k}=\sum e_{i} \gamma_{j k i}, & M_{2} \cdot e_{j} e_{k}=\sum e_{i} \gamma_{k j i}, & M_{3} \cdot e_{j} e_{k}=\sum e_{i} \gamma_{j i k}, \\
M_{4} \cdot e_{j} e_{k}=\sum e_{i} \gamma_{i k j}, & M_{5} \cdot e_{j} e_{k}=\sum e_{i} \gamma_{k i j}, & M_{6} \cdot e_{j} e_{k}=\sum e_{i} \gamma_{i j k} .
\end{array}
$$

If we compare these with the entries in the array of $X$ 's, we find that we can deduce one set of arrays from the other and reciprocally. In all the cases we change $x_{i}$ to $e_{i}$ and vice versa. We thus arrive at the simple rules following:

To derive the determinant of each algebra for the general number we change $e$ to $x$ in the multiplication table indicated, deriving $\Delta^{1}$ from $M_{6}, \Delta^{2}$ from $M_{3}$, $\Delta^{3}$ from $M_{4}, \Delta^{4}$ from $M_{2}, \Delta^{5}$ from $M_{1}, \Delta^{6}$ from $M_{5}$.

To derive the multiplication table of each algebra we change $x$ to $e$ in the determinant array indicated, deriving $M_{1}$ from $\Delta^{5}, M_{2}$ from $\Delta^{4}, M_{3}$ from $\Delta^{2}, M_{4}$ from $\Delta^{3}, M_{5}$ from $\Delta^{6}, M_{6}$ from $\Delta^{1}$.

5. The relationship of the $X$ 's to each other is seen more clearly if we use the notation of general vector algebra, ${ }^{*}$ which gives us each $X$ as an inner product:

$$
\begin{array}{lll}
X_{j k}^{1}=A_{0} e_{k}\left(\rho e_{j}\right), & X_{j k}^{2}=A_{0} e_{k}\left(e_{j} \rho\right), & X_{j k}^{3}=A_{0} e_{j}\left(\rho e_{k}\right), \\
X_{j k}^{4}=A_{0} \rho\left(e_{k} e_{j}\right), & X_{j k}^{5}=A_{0} \rho\left(e_{j} e_{k}\right), & X_{j k}^{6}=A_{0} e_{j}\left(e_{k} \rho\right) .
\end{array}
$$

Considering $\rho$ as an operator (linear substitution) we may write $\rho^{\prime}$ for the transverse operator and then these expressions become ( $k$ indicating columns, $j$ rows ) the following inner products:

$$
\begin{array}{lll}
X_{j k}^{1}=A_{0} e_{k}\left(\rho e_{j}\right), & X_{j k}^{2}=A_{0} e_{k}\left(e_{j} \rho\right), & X_{j k}^{3}=A_{0} e_{k}\left(\rho^{\prime} e_{j}\right), \\
X_{j k}^{4}=A_{0} e_{k}\left(\rho e_{j}^{\prime}\right), & X_{j k}^{5}=A_{0} e_{k}\left(e_{j}^{\prime} \rho\right), & X_{j k}^{6}=A_{0} e_{k}\left(e_{j} \rho^{\prime}\right) .
\end{array}
$$

6. From this notation we see that we may consider $\rho$ as a linear vector operator in each case. The respective determinants become $\dagger$

\footnotetext{
* $A_{0} \cdot \rho \sigma \equiv \Sigma x_{i} y_{i}$. This notation the author has used in other papers.

$\dagger A_{0} \cdot A_{s} \alpha_{1} \cdots \alpha_{s} A_{s} \beta_{1} \cdots \beta_{s}=(-1)^{\frac{1}{2} s(s-1)}\left|A_{0} \cdot \alpha_{i} \beta_{j}\right|, \quad(i, j=1, \cdots, s)$, $A_{0} \cdot A_{s} \alpha_{1} \cdots \alpha_{s} A_{s} \phi \beta_{1} \cdots \phi \beta_{s}=A_{0} A_{s} \phi^{\prime} \alpha_{1} \cdots \phi^{\prime} \alpha_{s} A_{s} \beta_{1} \cdots \beta_{s}$.
} 


$$
\begin{aligned}
& \Delta^{1}=t A_{0} A_{r} e_{1} e_{2} \cdots e_{r} A_{r}\left(\rho e_{1}\right)\left(\rho e_{2}\right) \cdots\left(\rho e_{r}\right), \\
& \Delta^{2}=t A_{0} A_{r} e_{1} e_{2} \cdots e_{r} A_{r}\left(e_{1} \rho\right)\left(e_{2} \rho\right) \cdots\left(e_{r} \rho\right), \\
& \Delta^{3}=t A_{0} A_{r} e_{1} e_{2} \cdots e_{r} A_{r}\left(\rho^{\prime} e_{1}\right)\left(\rho^{\prime} e_{2}\right) \cdots\left(\rho^{\prime} e_{r}\right), \\
& \Delta^{4}=t A_{0} A_{r} e_{1} e_{2} \cdots e_{r} A_{r}\left(\rho e_{1}^{\prime}\right)\left(\rho e_{2}^{\prime}\right) \cdots\left(\rho e_{r}^{\prime}\right), \\
& \Delta^{5}=t A_{0} A_{r} e_{1} e_{2} \cdots e_{r} A_{r}\left(e_{1}^{\prime} \rho\right)\left(e_{2}^{\prime} \rho\right) \cdots\left(e_{r}^{\prime} \rho\right), \\
& \Delta^{6}=t A_{0} A_{r} e_{1} e_{2} \cdots e_{r} A_{r}\left(e_{1} \rho^{\prime}\right)\left(e_{2} \rho^{\prime}\right) \cdots\left(e_{r} \rho^{\prime}\right),
\end{aligned}
$$

where $\left(t=(-1)^{r(r-1) / 2}\right)$.

We see from these forms at once that we have identically $\Delta^{3}=\Delta^{1}, \Delta^{6}=\Delta^{2}$, and, by writing out the determinants, $\Delta^{4}=\Delta^{5}$. Owing to the alternating character of the expressions $A_{r}$, they are not altered if we substitute $e_{i}+h e_{j}$ for $e_{i}$, where $h$ is any arbitrary scalar multiplier. But a repetition of similar substitutions would enable us to introduce a new basis, which we can represent by $\epsilon_{1}, \epsilon_{2}, \cdots, \epsilon_{r}$, where $\epsilon_{i}=\phi e_{i}, \phi$ being a linear substitution on the basis such that $|\phi| \neq 0$. That is to say, in place of each $e$ in the forms above we may write $\epsilon$; but it is to be noticed that the combinations with $\rho$ are still formed under the original multiplication table. If now we desire to consider $\rho$. as expressed in terms of the basis $\epsilon$, and also make our inner product $A_{0}$ refer to the new defining units, we must remember that if $A_{0}^{\prime}$ refers to the basis $\epsilon$ just as $A_{0}$ to the basis $e$,

$$
A_{0}^{\prime} \xi \zeta=A_{0} \xi \phi^{\prime-1} \phi^{-1} \zeta,
$$

where $\phi^{\prime}$ is the transverse of $\phi$. We will let $\phi \phi^{\prime}=\psi$, which is self-transverse. Then

$$
A_{0}^{\prime} \xi \zeta=A_{0} \xi \psi^{-1} \zeta .
$$

Also we notice that $A_{0}^{\prime} \xi \theta(\zeta)=A_{0}^{\prime} \zeta\left[\psi \theta^{\prime} \psi^{-1}(\xi)\right]$ and ( $) \rho^{\prime}$ for basis $\epsilon$ is $\psi\left[\left\{\psi^{-1}()\right\} \rho^{\prime}\right]$ for basis $e$; $\rho^{\prime}()$ for $\epsilon$ is $\psi\left\{\rho^{\prime}\left(\psi^{-1}[]\right)\right\}$ for $e$.

The expression $X_{j k}$ becomes under this change of basis ( $\rho$ now in terms of $\epsilon$ )

$$
{ }^{\prime} X_{j k}^{1}=A_{0}^{\prime} \epsilon_{k}\left(\rho \epsilon_{j}\right)=A_{0} \phi^{\prime-1} e_{k}\left(\rho \phi e_{j}\right)
$$

and thus the determinant in terms of $A_{0}^{\prime}$, which we may represent by ' $\Delta^{1}$, is ${ }^{\prime} \Delta^{1}=A_{0} A_{r} \phi^{\prime} e_{1} \phi^{\prime} e_{2} \cdots \phi^{\prime} e_{r} A_{r}\left(\rho \phi^{\prime-1} \epsilon_{1}\right)\left(\rho \phi^{\prime-1} \epsilon_{2}\right) \cdots\left(\rho \phi^{\prime-1} \epsilon_{r}\right)(-)^{\frac{1}{2} r(r-1)}$, which reduces to

$$
|\phi| \cdot\left|\phi^{-1}\right| \cdot \Delta^{1}=\Delta^{1}
$$

and it is evident that $\Delta^{1}$ is invariant under a change of basis.

It is obvious that corresponding changes in the other determinants will leave them also invariant.

7. Returning to $\S 2$ and using the last equations in $\S 5$, and since 
$\xi=\sum e_{k} A_{0} e_{k} \xi$, we see that we may define the six multiplications in terms of $\rho$ and the operator $\rho^{\prime}$ thus:

$$
\begin{array}{lll}
M_{1} \cdot \rho e_{j}=\rho e_{j}, & M_{2} \cdot \rho e_{j}=e_{j} \rho, & M_{3} \cdot \rho e_{j}=\rho^{\prime} e_{j}, \\
M_{4} \cdot \rho e_{j}=\rho e_{j}^{\prime}, & M_{5} \cdot \rho e_{j}=e_{j}^{\prime} \rho, & M_{6} \cdot \rho e_{j}=e_{j} \rho^{\prime} .
\end{array}
$$

When neither letter is accented we have the original strophic multiplication. The general formula of multiplication is then easily found by substituting the general number $\sigma$ for $e_{j}$. There is obviously no essential difference between an algebra and the anti-algebra save as to which of the two numbers we consider to be the multiplicand. We may further omit the multiplication symbol $M$, since the three essentially different types of multiplication are now indicated definitely by writing

$$
\rho \sigma, \quad \rho^{\prime} \sigma, \quad \rho \sigma^{\prime},
$$

which are respectively the strophic, preparastrophic, postparastrophic, for $\rho$ into $\sigma$, and are the corresponding anti-algebra for $\rho$ by $\sigma$.

8. When the strophic is associative, we have

$$
\rho \cdot \sigma \cdot()=(\rho \sigma) \cdot(), \quad \rho^{\prime} \cdot \sigma^{\prime} \cdot()=(\sigma \cdot \rho \cdot)^{\prime}()=(\sigma \rho)^{\prime}(),
$$

with similar equations for the post multiplication. Hence

$$
(\rho \eta)^{\prime} \xi=\eta^{\prime} \rho^{\prime} \xi \text { or }[() \eta]^{\prime} \xi=\eta^{\prime}\left[()^{\prime} \xi\right] .
$$

Therefore we have the following equation in the determinants:

$$
\Delta^{5}(\xi) \cdot \Delta^{2}(\eta)=\Delta^{3}(\eta) \cdot \Delta^{5}(\xi) .
$$

We might equally well have written $\xi[\eta()]^{\prime}=\xi()^{\prime} \cdot \eta^{\prime}$ whence

$$
\Delta^{4}(\xi) \cdot \Delta^{1}(\eta)=\Delta^{6}(\eta) \cdot \Delta^{4}(\xi) .
$$

It follows at once when $\Delta^{4}(\xi)=\Delta^{5}(\xi) \neq 0$ that we have identically

$$
\Delta^{1}(\eta)=\Delta^{3}(\eta)=\Delta^{2}(\eta)=\Delta^{6}(\eta) .
$$

Further we have easily

and at once

$$
\rho^{\prime}\left(\eta^{\prime} \xi\right)=(\eta \rho)^{\prime} \xi \text { or }()^{\prime}\left(\eta^{\prime} \xi\right)=[\eta()]^{\prime} \xi,
$$

Also

$$
\Delta^{5}\left(\eta^{\prime} \xi\right)=\Delta^{5}(\xi) \cdot \Delta^{1}(\eta)=\Delta^{4}\left(\eta^{\prime} \xi\right)=\Delta^{2}(\eta) \cdot \Delta^{4}(\xi) .
$$

giving

$$
\xi \eta^{\prime} \rho^{\prime}=\xi(\rho \eta)^{\prime} \text { or } \quad\left(\xi \eta^{\prime}\right)()^{\prime}=\xi[() \eta]^{\prime},
$$

$$
\Delta^{4}\left(\xi \eta^{\prime}\right)=\Delta^{\prime}(\xi) \Delta^{2}(\eta)=\Delta^{4}\left(\eta^{\prime} \xi\right) .
$$

These equations express Frobenius' fundamental theorems.

9 . The general equation of the number $\rho$, whether for pre- or post-multi- 
plication, has certain irreducible factors of determinant form corresponding to the invariant regions of $\rho$. Let one of these be $\Phi$ corresponding to an invariant region of order $s$, then we can also write

$$
\Phi=\rho^{s}-m_{1} \rho^{s-1}+m_{2} \rho^{s-2}-\cdots(-)^{s} m_{s} e_{1}
$$

and for a certain set of $s$ units $e_{2}, \cdots, e_{s+1}$ we have for premultiplication,

$$
\Phi e_{i}=0, \quad i=2, \cdots, s+1,
$$

and for a certain set of $s$ units $a_{2}, \cdots, a_{s+1}$ we have $a_{i} \Phi=0$.

The coefficient $m_{s}$ may then be written in the form

$$
\begin{aligned}
m_{s} & =A_{0} A_{s}\left(\rho e_{2}\right)\left(\rho e_{3}\right) \cdots\left(\rho e_{s+1}\right) A_{s} e_{2} e_{3} \cdots e_{s+1}(-)^{\frac{1 s(s-1)}{s}} \\
& =A_{0} A_{s}\left(a_{2} \rho\right)\left(a_{3} \rho\right) \cdots\left(a_{s+1} \rho\right) A_{s} a_{2} a_{3} \cdots a_{s+1}(-)^{\frac{1 s(s-1)}{s}} .
\end{aligned}
$$

We will now operate upon both these with the generalized Hamiltonian operator* $\nabla$, giving

$$
\begin{aligned}
& \nabla m_{s}=\sum_{t=2}^{s+1} A_{1} A_{s-1}\left(\rho e_{2}\right) \cdots(i) \cdots\left(\rho e_{s+1}\right) A_{s} e_{2} e_{3} \cdots e_{s+1} \cdot(-)^{\frac{1 s(s-1)+i}{2}} \cdot e_{i}^{\prime} \\
& =\sum a_{i}^{\prime} A_{1} A_{s-1}\left(a_{2} \rho\right) \cdots(i) \cdots\left(a_{s+1} \rho\right) A_{s} a_{2} a_{3} \cdots a_{s+1} \cdot(-)^{s^{s(s-1)+i}} \\
& =\sum_{i} \sum_{t} e_{t} e_{i}^{\prime} A_{0} A_{s-1}\left(\rho e_{1}\right) \cdots(i) \cdots\left(\rho e_{s+1}\right) A_{s-1} e_{2} \cdots(t) \\
& \cdots e_{s+1}(-)^{\frac{1}{8}(s-1)} \\
& =\sum_{i} \sum_{t} e_{i}^{\prime} e_{t} A_{0} A_{s-1}\left(a_{2} \rho\right) \cdots(i) \cdots\left(a_{s+1} \rho\right) A_{s-1} a_{2} \cdots(t) \\
& \cdots a_{s+1}(-)^{\frac{1 s(s-1)}{s(n)}} \text {. }
\end{aligned}
$$

The $(i)$ indicates an omitted factor.

Multiplying postparastrophically and preparastrophically into $\Phi$ we have, since $\Phi e_{i}=0=a_{i} \Phi$,

$$
\nabla m_{s} \Phi^{\prime}=0=\Phi^{\prime} \nabla m_{s} .
$$

Again, since for any number $\sigma$ the first scalar invariant in the field that $\Phi$ annuls is given by

$$
\begin{aligned}
(-)^{\frac{1}{3} s(s-1)} m_{1}^{(s)}=A_{0} A_{s}\left(\sigma \lambda_{1}\right) \cdots & \lambda_{s} A_{s} \lambda_{1} \cdots \lambda_{s} \\
& +A_{0} A_{s} \lambda_{1}\left(\sigma \lambda_{2}\right) \cdots \lambda_{s} A_{s} \lambda_{1} \cdots \lambda_{s}+\cdots
\end{aligned}
$$

where the numbers $\lambda$ are linearly independent, we have $A_{0}(\sigma \rho) \nabla m_{s}=m_{1}^{(s)}(\sigma) m_{s}^{(s)}=A_{0}(\rho \sigma) \nabla m_{s}^{(s)}=A_{0} \sigma\left(\Delta m_{s}^{(s)} \rho^{\prime}\right)=A_{0} \sigma\left(\rho^{\prime} \nabla m_{s}^{(s)}\right)$. Now $m_{1}(\sigma)$ is linear in the coefficients of $\sigma$ and we may therefore set

$$
m_{1}^{(s)}(\sigma)=A_{0} \sigma \kappa_{s}, \quad \kappa_{s}=\sum e_{i} m_{1}^{(s)}\left(e_{i}\right),
$$

${ }^{*} \nabla \equiv \Sigma e_{i} \frac{\partial}{\partial x_{i}}$. 
and the numbers $\kappa$ so defined for the different values of $s$ are constant for the basis $e$. They usually vary for the change of basis. Making this substitution and remembering that $\sigma$ is any number we have

$$
m_{s}^{(s)} \kappa_{s}=\nabla m_{s}^{(s)} \rho^{\prime}=\rho^{\prime} \nabla m_{s}^{(s)}, \quad \text { or } \quad \kappa_{s}\left(m_{s}^{(s)} \rho^{\prime-1}\right)=\nabla m_{s}^{(s)} .
$$

It is to be remembered that as the numbers $\lambda$ were linearly independent this equation holds for those values of $\rho$ that make $\rho e_{2}, \rho e_{3}, \cdots, \rho e_{s+1}$ linearly independent. In such case $m_{s}^{(s)} \neq 0$, and we have

$$
\kappa_{s}=\nabla \log m_{s}^{(s)} \rho^{\prime}=\rho^{\prime} \nabla \log m_{s}^{(s)} .
$$

By parastrophic multiplication we see that we have also

$$
m_{s}^{(s)} \kappa_{s} \xi^{\prime}=\nabla m_{s}^{(s)}(\xi \rho)^{\prime}, \quad m_{s}^{(s)} \xi^{\prime} \kappa_{s}=(\rho \xi)^{\prime} \nabla m_{s}^{(s)} .
$$

10. If we multiply the different $\kappa$ 's for the $p$ different irreducible factors of the general equation by arbitrary constants $c$ and add we have

$$
\sum c_{s} \kappa_{s}=\rho^{\prime} \nabla \log \left(m_{s_{1}}^{(1)^{c_{1}}} \cdot m_{s_{2}}^{(2)^{c_{2}}} \cdots m_{s_{p}}^{(p)^{c_{p}}}\right) .
$$

This cannot vanish identically for arbitrary values of the constants without the product in the parenthesis being constant in the coefficients of $\rho$, which is absurd. Hence the different numbers $\kappa$ are linearly independent.

What has been said above for certain irreducible factors of the general equation will hold equally well for products of these factors, no factor being repeated and for the regions corresponding. If however we study an irreducible factor raised to a power not higher than its power in the general prelatent equation, or the general postlatent equation, we see from the formula above that we have no new $\kappa$ but simply a multiple of the original $\kappa$, which multiple is $\kappa$ times the power of the irreducible factor. A similar statement will hold for the numbers deduced from combinations of the irreducible factors, and we find thus combinations of the form $\sum \mu_{s} \kappa_{s}$, where the numerical values $\mu$ are the indices of the powers of the factors. When these are the indices in the general prelatent equation we arrive at a special number we will call $\kappa_{1}$. It is evident that

$$
\kappa_{1}=\sum e_{i} m_{1}^{\prime}\left(e_{i}\right)=\sum A_{0} e_{j}\left(e_{i} e_{j}\right) \cdot e_{i}=\sum e_{j} e_{j}^{\prime} .
$$

There is likewise a number arising from the postlatent equation which will be called $\kappa_{2}$,

$$
\kappa_{2}=\sum e_{i} m_{1}^{\prime \prime}\left(e_{i}\right)=\sum e_{i} A_{0} e_{j}\left(e_{j} e_{i}\right)=\sum e_{j}^{\prime} e_{j}
$$

From these we have easily

$$
m_{1}^{\prime}(\rho)=A_{0} \rho \kappa_{1}, \quad m_{1}^{\prime \prime}(\rho)=A_{0} \rho \kappa_{2} .
$$


Further $\kappa_{1}=\nabla \rho^{\prime}, \kappa_{2}=\rho^{\prime} \nabla$. We have in a manner entirely similar to the process above

$$
\kappa_{1}=\nabla \log m_{r}^{\prime} \rho^{\prime}, \quad \kappa_{2}=\rho^{\prime} \nabla \log m_{r}^{\prime \prime} .
$$

These forms for $\kappa_{1}, \kappa_{2}$ are particularly simple. Setting the expressions for $\kappa_{1}$ equal and those for $\kappa_{2}$ equal we have easily

$$
\nabla\left(\rho^{\prime} / m_{r}^{\prime}\right)=0=\left(\rho^{\prime} / m_{r}^{\prime \prime}\right) \nabla .
$$

We cannot assume from these that the expressions in the parentheses are constants for the vanishing is due partly to the hypercomplex multiplication and not wholly to the differentiation.

From the definition we see that if we change the basis we have

$$
A_{0} \sigma \kappa=A_{0}^{\prime} \sigma \psi \kappa=A_{0}^{\prime} \sigma \kappa_{\epsilon},
$$

where $\kappa_{\mathrm{e}}$ signifies that we find the number $\kappa$ for the new basis. Comparing these equations we have at once $\kappa_{\epsilon}=\psi_{\kappa}$, since $\sigma$ is any number. This equation holds for all the numbers $\kappa$.

11. It is clear from the last equations in $\S 9$ that the only number that will give $\kappa \xi^{\prime}=\kappa$ or $\xi^{\prime} \kappa=\kappa$, for any given irreducible factor, will be the number for which for that latent region $\rho \xi=\rho=\xi \rho$ for all values of $\rho$ in the region which have a non-vanishing $m$, that is it will be the partial modulus of the latent region. Again, since $\nabla m_{\dot{s}} \Phi^{\prime}=0$, it follows that also

$$
\kappa_{s} \Phi^{\prime}=0=\Phi^{\prime} \kappa_{s} .
$$

The number $\kappa$ is thus entirely within the $s$ th latent region of $\rho^{\prime}$, either pre or post.

12. It is evidently an interesting question to answer as to when $\rho^{\prime}$ is itself not simply an operator determined by $\rho$, but a number of the system. If we let the semi-simple subalgebra of $A$ be $B$ and the maximal invariant nilpotent system be $N$, then $A=B+N$. Now let us suppose that $\rho$ is a number in $N$ and has a transverse $\rho^{\prime}=\tau$ in the algebra $A$. Then since the product of $\rho$ into any number $\xi$ of the system is in $N$, we have if $\beta$ is in $B$

$$
A_{0} \beta(\rho \xi)=0=A_{0} \xi\left(\rho^{\prime} \beta\right)=A_{0} \xi(\tau \beta) \quad \text { (for any } \xi \text { ). }
$$

Hence $\tau \beta=0$, for $\beta$ in $B$. But the modulus $e_{1}$ is in $B$ and thus $\tau e_{1}=\tau=0$, and therefore $A_{0} \beta(\tau \xi)=0=A_{0} \xi(\rho \beta)$, and $\rho=0$.

13. It follows at once that if every number of the algebra has a transverse in the algebra, then the algebra has no maximal invariant nilpotent system and is a Dedekind algebra.

This furnishes an extremely simple proof that every finite group defines a Dedekind algebra of a special kind, a Frobenius algebra. For if the operators 
of the group are represented by $e_{1}, \cdots e_{n}$, then we may represent the product $e_{i} e_{j}$ by $e_{i j}$; and if $\rho=\sum x_{i} e_{i}$ we have

$$
\begin{gathered}
A_{0} \sum z_{i} e_{i}\left(\sum x_{j} e_{j} \sum t_{k} e_{k}\right)=A_{0} \sum z_{i} e_{i}\left(\sum x_{j} t_{k} e_{j k}\right)=\sum z_{j k} x_{j} t_{k} \\
A_{0} \sum t_{k} \epsilon_{k}\left(\sum x_{j} \epsilon_{j}^{-1} \sum z_{i} e_{i}\right)=A_{0} \sum t_{k} e_{k} \sum x_{j} z_{i} e_{j i}^{-1}=\sum z_{i} x_{j} t_{j i}^{-1}=\sum z_{j k} x_{j} t_{k} .
\end{gathered}
$$

It is obvious then that the transverse of $\rho=\sum x_{i} e_{\imath}$ is in the algebra and is

$$
\tau=\rho^{\prime}=\sum x_{i} e_{i}^{-1} .
$$

Likewise in the semi-simple system let the units be $\lambda_{p q}$ where $\lambda_{p q} \lambda_{r s}=\delta_{q r} \lambda_{p s}$, the symbol $\delta_{q r}$ having the value 0 if $q \neq r$, and 1 if $q=r$. Then we have

$$
\begin{aligned}
A_{0} \sum z_{p q} \lambda_{p q}\left(\sum x_{u v} \lambda_{u v} \sum t_{g h} \lambda_{g h}\right) & =A_{0} \sum z_{p q} \lambda_{p q}\left(\sum x_{u v} t_{v h} \lambda_{u h}\right)=\sum z_{u h} x_{u v} t_{v h}, \\
A_{0} \sum t_{g h} \lambda_{g h}\left(\sum x_{u v} \lambda_{u v} \sum z_{p q} \lambda_{p q}\right) & =A_{0} \sum t_{g h} \lambda_{g h}\left(\sum x_{p v} z_{p q} \lambda_{v q}\right) \\
& =\sum t_{v q} x_{p v} z_{p q}=\sum z_{u h} x_{u v} t_{v h} .
\end{aligned}
$$

Thus the transverse of $\rho$ exists and is

$$
\rho^{\prime}=\tau=\sum x_{v u} \lambda_{u v} .
$$

It would be interesting to apply to these two examples the preceding theorems, but we will content ourselves only with pointing out that for the group

$$
\kappa_{1}=\nabla \rho^{\prime}=\sum e_{i} e_{i}^{-1}=n e_{1}=\kappa_{2},
$$

that is, $n$ times the identical operator. For the semi-simple or Dedekind algebra

$$
\kappa_{1}=\kappa_{2}=\nabla \rho^{\prime}=\sum \lambda_{v u} \lambda_{u v}=\sum w_{j} \eta_{j},
$$

where $w_{j}^{2}$ and $\eta_{j}$ are respectively the orders of the integral subalgebras and their partial moduli.

14. If the basis of the algebra is subjected to the non-singular transformation $\phi$, and $\phi \phi^{\prime}=\psi$, we have seen already that any operator $\theta^{\prime}$ becomes $\psi \theta^{\prime} \psi^{-1}$, hence as operator, $\rho^{\prime}$ on the new basis would be $\psi \rho^{\prime} \psi^{-1}$ in terms of the old basis. Hence the transverse of $\rho$ on the new basis in terms of the units of the old basis is not $\tau$ but becomes

$$
\tau_{e}=\psi\left[\tau \psi^{-1} e_{1}\right]
$$

where $e_{1}$ is the modulus, and $\tau_{\epsilon} \sigma=\psi\left[\tau \psi^{-1} \sigma\right]$. Hence

$$
\tau_{\epsilon} \psi e_{1}=\psi \tau, \quad \tau_{\epsilon} \psi e_{i}=\psi\left(\tau e_{i}\right) .
$$

These equations are not satisfied for every value of $\phi$, nor $\psi$, but only for those values which permit of the simultaneous existence of the equations. In other words, if we let $\tau$ run through the entire range of numbers in the algebra we must have in all cases as a condition on $\psi$

$$
\psi \tau=\tau_{\epsilon} \psi e_{1}=\psi\left(\tau \psi^{-1} e_{1}\right) \psi e_{1}=\psi e_{1}\left(\psi^{-1} e_{1} \cdot \tau\right) .
$$


We may state thus the condition on $\psi$ from this equation: If we form the multiplication table for the original basis, and then operate on multipliers, multiplicands, and products all alike by either $\left(\psi e_{1}\right)^{-1} \psi()$ or $\psi()\left(\psi e_{1}\right)^{-1}$ the new table must be still a correct multiplication table for the algebra.

It is to be noticed that $\psi e_{1}$ is a multiple of $\kappa_{1}=\kappa_{2}$ for the new basis. It is also evident that $\kappa=\kappa_{1}=\kappa_{2}$ must have an inverse. Any operator $\phi$ which leads to $\psi$ such that $\psi e_{1}=\kappa$ does not have an inverse, is not permissible. The same algebra may therefore be such that it permits a transverse for every number on some bases but not on others.

15. When $\rho^{\prime}=\tau$ is in the algebra the preparastrophic and the postparastrophic multiplications can be defined in terms of the strophic multiplication.

For example, in the case of an abstract group, parastrophic multiplication by $\rho$ is strophic multiplication by $\sum x_{i} e_{i}^{-1}$.

UNIVERISTY OF ILLINOIS. 